Cahiers de recherches médiévales

\title{
Saül et ses démons
}

Un personnage biblique à la croisée des discours, de la fin du Moyen Âge à la Renaissance

\section{Bruno Méniel}

\section{(2) OpenEdition \\ 1 Journals}

Édition électronique

URL : https://journals.openedition.org/crm/770

DOI : $10.4000 / \mathrm{crm} .770$

ISSN : 1955-2424

Éditeur

Honoré Champion

\section{Édition imprimée}

Date de publication : 30 décembre 2006

Pagination : 221-236

ISSN : $1272-9752$

\section{Référence électronique}




\section{rin}

\section{Saül et ses démons : un personnage biblique à la croisée des discours, de la fin du Moyen Âge à la Renaissance}

Le Moyen Âge, puis la Renaissance ont prisé le raisonnement par cas : à partir d'une situation saisie dans sa singularité, s'élabore une réflexion dont les conclusions ont une portée générale. Ainsi lorsque les grandes figures de la Bible ne sont pas interprétées par allégorie, elles peuvent être appréhendées comme des cas. Le personnage de Saül est à ce titre exemplaire, parce qu'il apparaît dans trois discours spécialisés, celui des théoriciens politiques, celui des médecins et celui des théologiens ; il a, de plus, retenu l'attention des dramaturges. En effet, de cet homme tourmenté émane un mystère: quel est le mal qui le ronge? Aux $\mathrm{XV}^{\mathrm{e}}$ et $\mathrm{XVI}^{\mathrm{e}}$ siècles, les textes manifestent des signes, qui suggèrent des causes : le despotisme, la manie ou la mélancolie, l'irascibilité ou l'envie, le démon... Cette étiologie peut paraître hétéroclite. Notre hypothèse de départ est qu'il n'en est rien et que la multitude des figures de Saül serait médiatisée par un système de représentation qui assurerait la coexistence harmonieuse de plusieurs caractéristiques: les interprétations du mal de Saül ne se contrediraient pas, elles se cumuleraient et même, jusqu'à un certain point, elles s'agenceraient logiquement.

Nous considérerons d'abord les différentes interprétations que les textes donnent à la colère de Saül; nous examinerons ensuite comment ces significations s'articulent entre elles; enfin, à partir de cette étude, nous proposerons quelques remarques sur l'interpénétration des savoirs et la perméabilité des discours à la fin du Moyen Âge et à la Renaissance.

Saül se définit d'abord par sa fonction historique et sa colère peut apparaître comme un indice de despotisme. Les sages d'Israël, lassés par la corruption des fils de Samuel, ont demandé un roi. Désigné par Dieu, Saül est oint par Samuel, et les théoriciens de la monarchie française insistent sur l'efficace de l'onction, qui se vérifie aussi bien dans le cas de Saül que dans celui des rois de France. L'onction céleste signale que la divine providence élit les défenseurs de la loi sainte :

[...] Samuel print une phiole d'huyle, et l'espandit sur la teste de Saul, puis le baisa et dict : Le Seigneur ne t'a il pas oinct pour estre prince sur son heritaige ? [I S 10, 1] Voyez comme le prophete dit, que c'est Dieu mesmes qui a oinct le Roy. [...] Saul, [...] apres avoir esté oinct par Samuel, incontinent qu'il eut tourné le dos pour s'en aller d'avec Samuel, Dieu luy mua son cueur en un autre ${ }^{1}$.

L'onction change Saül "en un autre homme ${ }^{2} »$ : elle le fait passer de l'ordre du profane à celui du sacré. Cette mutation est commentée par les «miroirs du prince». Par exemple un ouvrage traditionnel, l'Institution du Prince chestien, de Jean

\footnotetext{
${ }^{1}$ Gabriel de Saconay, De la Providence de Dieu sur les rois de France très chrestiens [...], Lyon, Michel Jove, 1568, p. 9-10.

${ }^{2}$ I S $10,6:$ in virum alium ».
}

Cahiers de Recherches Médiévales, 13, 2006 
Talpin, invoque l'exemple de Saül pour prouver que «les Rois par la vertu de leur onction ont changé de naturels ${ }^{3} »$ :

\begin{abstract}
Aussi tost que Saül est oingt Roy, il Prophetise entre les Prophetes, disant merveilles, recitant et chantant les louanges de Dieu : il est faict bien tost sçavant, luy qui estoit auparavant fort rude et ignorant en la loy de Dieu : Il faict incontinent la guerre contre les Ammonites, Philistins, et Amalechites idolatres, ainsi qu'il avoit commandement de Dieu de ce faire : et le faict de cœur hardy et magnanime, luy qui avoit esté, avant qu'estre Roy, si timide et couart, qu'il n'eust osé desgaigner un couteau contre son mortel ennemy : et en tous assaults de la bataille, il vainquit ses ennemis par memorables victoires, au commencement de son regne : car aussi il faisoit telles entreprises selon le vouloir et commendemens de Dieu ${ }^{4}$.
\end{abstract}

Saül marque bien le passage de la théocratie à la monarchie, mais celle-ci n'est pas indépendante du contrôle divin. Or Saül a le malheur de désobéir au Seigneur. Dès lors, sa vie chavire, son destin est brisé. Avant cette fêlure irrémédiable, Saül était l'élu du Seigneur, le chef militaire victorieux, le sauveur de la nation; après, David, consacré par Samuel alors qu'il n'est encore qu'un enfant, commence son ascension, et Saül n'est plus que le roi en titre, il perd sa légitimité réelle, il accumule les revers militaires: son statut est désormais équivoque et inconfortable. Le cycle des Mystères de la procession de Lille, représentés aux $\mathrm{XV}^{\mathrm{e}}$ siècle, manifeste bien cette rupture. Saül est le protagoniste de trois pièces, Le Sacre de Saül, La Guerre de Saül contre Amaleq, L'Attentat de Saül contre David. L'évolution du personnage de la première à la deuxième est saisissante. Dans Le Sacre de Saül, les lamentations des messagers de Jabès, qu'assiège le tyran Naas, transforment Saül et en font un chef brave et vertueux, animé d'une juste colère :

lors

se imprima en son ame et cors,

meismes au parfont de son ceur,

l'esperit de Nostre Seigneur ;

c'est-à-dire que meü fu

de force, constance et vertu.

Aussy fut tourblé et yré,

non point de ire a la verité

qui fust vicïeuse et dampnable,

mais de ire tresreconmandable,

comme ire d'amour et de desir

qu'il avoit de tost subvenir

et soy exposer corps et biens

a l'aÿde des citoiiens

de Jabès et a leur deffence.

Magnanimité d'excellence,

tu fus lors en Saül trouvee ${ }^{5}$.

3 J. Talpin, Institution du Prince chestien, Paris, Nicholas Chesneau, 1567, f. $14 \mathrm{v}^{\circ}$, manchette.

${ }^{4}$ Ibid., f. $14 \mathrm{r}^{\circ}-\mathrm{v}^{\circ}$.

${ }^{5}$ Le Sacre de Saül, v. 241-257, in Les Mystères de la procession de Lille, éd. A. Knight, t. II, Genève, Droz, 2003, p. 219. 
Dans La Guerre de Saül contre Amaleq, un basculement s'opère : si Saül victorieux peut encore être loué par un de ses soldats comme «le roy cremu et redoubté / en tout honneur, gloire et prosperité, tant exauchié de Dieu omnipotent ${ }^{6}{ }^{\prime}$, Samuel ne tarde pas à lui faire part du désaveu divin. La pièce s'efforce de justifier celui-ci par toute une série de péchés dont Saül est coupable : l'avarice (v. 552), l'ambition (v. 735), l'arrogance (v. 736), la présomption (v. 647), l'outrecuidance (v. 735). La conséquence de tous ces péchés est la désobéissance (v. 733), qui conduit Samuel à rapprocher Saül du sorcier et de l'idolâtre :

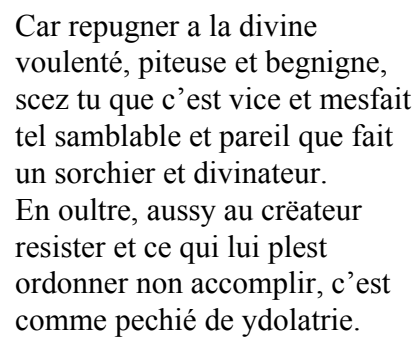

Ainsi, Les Mystères de la procession de Lille relèguent au second plan la question politique et se concentrent sur la relation entre éthique et théologie. Au XVI ${ }^{\mathrm{e}}$ siècle, en revanche, on perçoit plus nettement que les livres de Samuel tiennent un discours sur la monarchie, et on se rappelle que le prophète, dans un long discours ${ }^{7}$, met le peuple d'Israël en garde contre les abus des rois. Les protestants, les «monarchomaques» en particulier, s'emparent de la figure de Saül: premier roi d'Israël, il en est aussi le premier tyran. C'est ainsi que le caractérisent le Theatrum historicum du théologien Andreas Hondorff, publié pour la première fois en allemand en 1568, lorsqu'il commente l'épisode du massacre des prêtres de $\mathrm{Nov}^{8}$, et les Vindicia contra Tyrannos, imprimées pour la première fois en $1579^{9}$. Saül est un tyran parce qu'il a persécuté les hommes de Dieu et qu'il a opprimé David. La perspective strictement morale n'est pas pour autant abandonnée. Saül est aussi un tyran parce qu'il se met en colère : à la Renaissance, ce signe distingue le tyran du bon roi. De fait, Saül est qualifié d'iratus ${ }^{10}$ par le texte de la Vulgate. À plusieurs reprises, il perd le contrôle de lui-même. Il s'emporte contre d'autres que David, et notamment contre son fils Jonathan. Le Theatrum historicum fait de lui un exemple de «la colère et la sévérité des parents à l'égard des enfants ${ }^{11}$ », en s'appuyant sur

\footnotetext{
${ }^{6}$ La Guerre de Saül contre Amaleq, v. 431-433, in id., p. 279.

${ }^{7}$ I S 8, 11-18.

${ }^{8}$ I S 22, 6-23.

${ }^{9}$ Étienne Junius Brutus, Vindicia contra Tyrannos (1579), 1589, p. 203 : «Sauli tyranno» (trad. fr., De la puissance legtime du Prince sur le peuple, et du peuple sur le prince (1581), reprint Genève, Droz, 1979, p. 239 : « le tyran Saul »).

${ }^{10}$ Voir notamment I S 18,$8 ; 20,7$ et 30 .

${ }^{11}$ Andreas Hondorff, Theatrum historicum siue Promptuarium illustrium exemplorum (1568), trad. lat. P. Lonicer, Francfort, Johannes Bringer, 1616, p. 336.
} 
l'épisode où Saül reproche à Jonathan d'avoir transgressé un interdit en mangeant du miel $^{12}$.

Saül est donc utilisé par le pouvoir religieux pour rappeler aux monarques qu'ils ne sauraient s'affranchir de la tutelle divine.

L'irritation peut ensuite révéler le tempérament de Saül. Suffit-elle à faire de lui un colérique? Non : dans la médecine de l'époque, elle n'est pas nécessairement le symptôme d'une la prédominance de la bile jaune. Comme le remarque Jean Wier $^{13}$, il existe des colères propres à chaque tempérament; le sanguin, le flegmatique, le mélancolique s'irritent chacun à sa façon ${ }^{14}$. Saül est invariablement rangé parmi les mélancoliques. Dans Les Mystères de la procession de Lille, David déclare jouer de la harpe "Pour tenir le roy liiement / et hoster de melancolie ${ }^{15}$ ». Dans Le Mistère du viel Testament, Saül déclare :

Je suis plein de merencolie,

Si fort, que je ne sçay que dire ;

Je ne sçay se c'est par follie

Que je suis provoqué a ire ${ }^{16}$.

et il se plaint du trouble que ce mal suscite en lui :

Je ne sçay qui, dyable, me tient,

Mais je suis tresfantastique,

Si perplex et merencolique

Que j'ai l'entendement cassé.

Qui est-ce qui m'a ce brassé ${ }^{17}$ ?

Gilbert Genebrard accompagne de notes sa traduction des Antiquitez judäques de Flavius Josèphe, parue en 1578. Or, à propos de l'épisode où Saül est destitué de l'esprit de Dieu, il précise :

\footnotetext{
${ }^{12}$ I S $14,25-45$

${ }^{13}$ J. Wier, De Ira morbo, ejusdem curatione Philosophica, Medica et Theologica, Liber, Bâle, Ex officina Oporiniana, 1577, p. 17-18: "Verum hoc loco repudiari debet inueteratus multorum error, qui materialem irce caussam putant solam bilem, adeo ut apud Latinos bilis pro ira, et biliosus pro iracundo usurpetur, eadem licentia, qua apud Gracos cholè et cholerikos. » (En vérité, il convient ici de réfuter une erreur que commettent depuis longtemps de nombreux auteurs qui pensent que la seule cause matérielle de la colère est la bile, au point que chez les Latins, on emploie «bile » pour « colère » et « bilieux » pour « irascible », avec la même liberté que chez les Grecs, cholè et cholerikos.)

${ }^{14}$ Ibid., p. 17-22.

${ }^{15}$ L'Attentat de Saül contre David, v. 277-278, in Les Mystères de la procession de Lille, éd. cit., p. 318.

${ }^{16}$ Mistère du viel Testament, éd. J. de Rothschild, Paris, Firmin Didot, 1882, t. IV, v. 3052530528 .

${ }^{17}$ Ibid., v. 29656-29660.
} 
Les Rabbins n'entendent pas par l'esprit malin qui tourmentoit Saul un esprit qui le rendoit demoniaque, ains un esprit triste, et melancolique, qui le rendoit fasché et despaisant en soymesme ${ }^{18}$.

Saül finit par se donner la mort, durant la bataille de Guilboa, alors que les Philistins l'ont traqué et que ses trois fils ont été tués. Bien sûr, le choix du suicide s'explique par ces circonstances particulières, mais il peut aussi être attribué à la mélancolie. Voici comment, selon le moine Gaspar Ofhuys (1456-1523), les ecclésiastiques, soignaient un mélancolique, le peintre Hugo van der Goes, aux alentours de 1480 :

[...] sans cesse il se disait damné et voué à la damnation éternelle, et il voulait se donner réellement la mort (ce qu'il aurait fait s'il n'en avait été empêché de force par l'intervention des témoins) [...]. Il fut emmené sous bonne protection à Bruxelles, et sans retard, le Père supérieur Thomas est appelé ; après enquête et examen, il estima que le patient était agité du même mal que Saül. Et se rappelant comment Saül était soulagé quand David sonnait de la cithare, il permit que l'on jouât sur le champ devant le frère Hugo de la musique en abondance, et qu'on lui présentât aussi d'autres spectacles récréatifs, par lesquels il comptait chasser les visions mentales ${ }^{19}$.

La Renaissance semble donc intégrer les tendances suicidaires au complexe de Saül.

La colère de Saül peut en outre être analysée à la lumière de la théologie morale. Dans la Somme théologique de Thomas d'Aquin, la colère a un statut à part. Elle est à la foi une passion spéciale et l'effet de nombreuses autres: tous les mouvements de la puissance irascible «ont pour terme la colère, qui est le plus éclatant de tous ${ }^{20}$ " et « la colère contient plusieurs passions, non à la manière dont le genre contient ses espèces, mais plutôt selon l'inclusion de la cause dans ses effets. ${ }^{21}$ Peut-être convient-il donc de chercher la cause de la colère de Saül dans un autre vice, l'envie. La glose de Nicolas de Lyre à I S 18, 6 signale que les chants de

\footnotetext{
${ }^{18}$ Flavius Josèphe, Des Antiquitez judaïques, VI, 9, trad. G. Genebrard [1578], Paris, R. Bertault, 1639, p. 204.

${ }^{19}$ Gaspar Ofhuys, "Originale Cenobii Rubeevallis in Zonia prope Bruxellam in Brabancia » (Bibliothèque Royale de Bruxelles, Msc II, 48017) éd. H. G. Sander, in «Beiträge zur Biographie Hugos van der Goes' und zur Chronologie seiner Werke » in Repertorium für Kunstwissenschaft, XXXV, (1912), p. $535:$ : [...] incessanter dicebat esse dampnatum et dampnationi eterne adiudicatim, quo etiam sibi ipsi corporaliter et letaliter (nisi violenter impeditus fuisset auxilio astantium) nocere volebat. [...] Qui tamen auxilio opitulante Bruxellam pervenerunt, et sine mora pater prior Tomas illuc demandatur, ille cuncta videns et audiens, suspicabatur eum eodem moprbo vexari, quo rex Saul agitabatur. Unde recordans quomodo Saul. Unde recordans quomodo Saul levius habebat [D]avid cytharam percutiente, permisit ibidem coram fratre Hugone melodiam fieri non modicam, sed et alia spectacula recreativa, quibus intendebat mentales fantasias repellere. »

${ }^{20}$ S. Th., I-II, q. 46, a. 1, sol. 1 : «ad iram terminantur omnes motus huius potentice, et inter alios ejus motus iste est manifestior $»$.

${ }^{21}$ Ibid., sol. 3 : «ira includit multas passiones, non quidem sicut genus species, sed magis secundum continentiam causa et effectus. »
} 
louange adressés à David pour sa victoire sur Goliath ont provoqué l'inuidia de Saül ${ }^{22}$. Dès ce moment, Saül regarde David non rectis oculis : "c'est en effet, dit Nicolas de Lyre, la façon qu'ont les envieux et les haineux de regarder ceux qu'ils haïssent de manière oblique ${ }^{23} »$. L'Attentat de Saül contre David s'accorde avec cette lecture : Saül éprouve de l'envie dès la victoire de David sur Goliath, qui attire au jeune homme la faveur populaire : «envie sy le contraindoit / qu'il desiroit tousjours sa mort» (v. 14-15). Le succès que remporte David sur les Philistins oblige le roi à réprimer sa passion, mais il ne peut l'empêcher de se manifester, ainsi que l'annonce le Prologue :

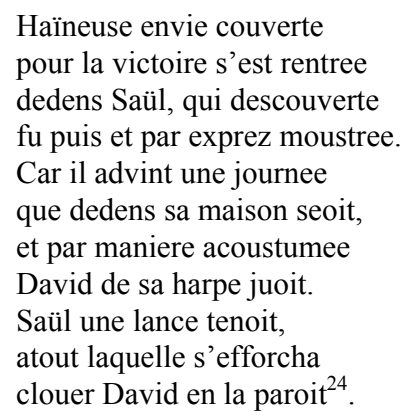

Les questions inquiètes que se pose Saül dans le Mistère du viel Testament, suggèrent l'envie qu'il éprouve envers David:

Verray je mon hault nom destruire?

Sera David regnant sur moy?

Me gardera il de reluyre?

M'ostera il le nom de roy?

Ha! David, David, c'est par toy

Que je suis en ceste tristesse ;

J'en ay au cueur si grant esmoy

Que pers tout plaisir et liesse ${ }^{25}$.

L'envie, absente des vices capitaux chez Cassien et Isidore, est introduite parmi eux par saint Grégoire dans ses Moralia in $I^{2} b^{26}$. Thomas d'Aquin, qui adopte le septenaire des vices de Grégoire, ajoute que l'envie est un des péchés les plus

\footnotetext{
${ }^{22}$ Voir Nicolas de Lyre, glose de I S 18, 6, Bible s.l.n.d. [1482 ?] disponible sur le serveur Gallica, f. cc4 r ${ }^{\circ}$.

${ }^{23}$ Id., glose de I S 18, 9, ibid. : «Modus est enim inuidorum et odientium respicere eos quos odiunt ex obliquo. »

${ }^{24}$ L'Attentat de Saül contre David, v. 261-271, Les Mystères de la procession de Lille, éd. cit., p. 317.

${ }^{25}$ Mistère du viel Testament, éd. J. de Rothschild, Paris, Firmin Didot, 1882, t. IV, v³052930536 .

${ }^{26}$ Grégoire le Grand, Moralia, XXXI, XVII, in Opera omnia qua extant, Froben, Bâle, 1584, t. I, col. 1082-1083.
} 
graves lorsqu'elle est suscitée par la grâce de nos frères ${ }^{27}$. Or Saül est précisément dans ce cas : délaissé par Dieu, il envie David d'avoir été élu, et c'est pourquoi il tente de le tuer:

De là Saül poursuit David qui lui est soumis en lui jetant une lance, parce qu'il a senti que chaque jour il était rehaussé pour les grands succès de ses vertus et qu'il a craint qu'il ne le dépasse ${ }^{28}$.

L'envie est à la fois un péché et une punition. Elle place Saül dans le cas où, selon la formule de Thomas d'Aquin, «le péché peut être une peine, par la substance même de son acte, à cause de l'affliction qu'il apporte ${ }^{29} »$. De plus, une des filles de l'envie est la dérision, dont la crainte, selon le pseudo-Vincent de Beauvais, peut pousser au suicide. Cela permettrait d'expliquer la fin que Saül s'est choisie :

Il faut se garder de ce vice à cause de l'angoisse d'être moqué. En effet, parfois la moquerie et les plaisanteries touchent davantage les hommes que les autres afflictions. Beaucoup ont préféré mourir que d'endurer les moqueries et les plaisanteries. Comme [...] Saül, duquel I Rois 31 : Saül dit à son écuyer : "Tue-moi, de peur que les incirconcis ne se moquent de moi $^{30}$."

Enfin, la colère de Saül peut être un châtiment divin ou un signe de possession démoniaque. Dans le Mistère du viel Testament, cette colère a pour origine celle du Tout-Puissant. Apparaissant sur scène, Dieu, qui a demandé à Saül, par l'intermédiaire de Samuel, d'exterminer Amalech, ses hommes et jusqu'à son bétail, s'irrite de voir qu'après avoir capturé le chef des ennemis héréditaires, il lui fait grâce. Dieu dit de Saül : «Courcé suis de l'avoir faict roy ${ }^{31}$ «. Or la punition qu'il lui inflige est précisément la colère : «Je le provoqueray à ire ${ }^{32}$ ». Dans les Tragédies saintes, Louis Des Masures fait paraître sur scène Satan, qui s'attribue tous les manquements aux ordres divins dont Saül s'est rendu coupable :

Sous ce pervers abus de cuider faire bien,

J'ai fait le Roy Saul, que sur le peuple sien

Dieu luy-mesme avoit mis, servir Dieu autrement

${ }^{27}$ S. Th., II-II, q. 36, a. 4, sol. 2.

${ }^{28}$ Grégoire le Grand, Moralia, V, XXXII, éd. cit., t. I, col. 175 : «Hinc Saul David subditum lanceam in torquendo persequitur, quia quem magnis quotidie augeri virtutum successibus sensit, ultra se excrescere expauit." Voir pseudo-Vincent de Beauvais, Bibliotheca mundi, seu Speculi maioris [...] tomus tertius, qui speculum morale inscribitur, Douai, Balthazar Bellère, 1624, col. 1141.

${ }^{29}$ S. Th., I-II, q. 87, a. 2, rép. : «peccatum dicitur esse pœna pracedentis peccati [...] ex parte substantice actus qui afflictionem inducit, [...] ut patet in ipsa ira et inuidia [...].»

${ }^{30}$ Pseudo-Vincent de Beauvais, op. cit., col. $1154:$ : [...] cauendum est hoc vitium propter derisi angustiam. Quandoque enim plus affligunt homines irrisiones et ludibria, quam alia afflictiones. Multi enim praelegerunt potius mori, quam derisiones et ludibria sustinere. Sicut [...] Saul, de quo I.Reg.31. Dixit Saul armigero suo: Interfice me, ne forte ueniant incircuncisi, et illudant mihi. »

${ }^{31}$ Mistère du viel Testament, éd. cit., t. IV, v. 29408.

${ }^{32}$ Ibid., v 29402. 
Que ne luy permettoit le divin mandement. Je l'ay fait en Galgal au sacrifice entendre, Et à son Dieu l'offrir, sans Samuel attendre, Comme enjoint luy estoit. J'ay fait qu'en Amalec Il a sauvé le Roy, et le butin d'illec,

Sans y garder de Dieu l'ordonnance et defense :

Combien qu'il estimast ne faire point offense,

Et que le gros bestail il eust intention

Sauver, pour puis apres en faire oblation

A son Dieu, qui des siens (sans qu'en soy on se fie)

Demande obeissance, et non qu'on sacrifie.

Parquoy il est de luy maintenant rejetté,

Privé de son esprit, et du Malin traitté ${ }^{33}$.

Que la fonction d'exposer la situation soit dévolue à Satan révèle que celui-ci mène le jeu. Par la reprise du verbe «faire» il montre qu'il manipule le roi d'Israël. Saül, dès sa deuxième réplique, se tourne vers Dieu pour lui reprocher de l'avoir mis sur le trône, pour ensuite le laisser à la merci des Philistins :

Tu as donques de moy retiré ton Esprit :

Tu m'as donc delaissé au malin, qui me prit,

Et qui vient m'agiter à tous coups d'une rage ${ }^{34}$.

Saül a péché par idolâtrie : ainsi, pour le pasteur George Gifford (1573-1620), lorsque Saül a fait appel aux services de la "sorcière d'Endor», il s'est voué au diable :

Ce n'est pas un petit crime que d'appeler le diable à son secours : c'est le mettre à la place de Dieu, et l'honorer comme Dieu. Cela vient de l'infidélité et de la perte de la foi en le secours divin, comme on le voit dans l'exemple du roi Saül, qui, ne recevant ni réponse ni réconfort de Dieu, à qui il avait si vilainement désobéi, alla chez une sorcière. Le païen dit: «Flectere si nequeo Superos, Acheronta movebo » [Én., VII, 312] $]^{35}$.

Chez les théologiens, le passage où il est dit que «L'esprit du Seigneur s'était retiré de Saül» et qu' «un esprit mauvais, venu du Seigneur, le tourmentait» (I S 16, 14) donne lieu à des commentaires qui insistent sur la soumission des démons à

${ }^{33}$ L. Des Masures, David combattant, v. 251-266, in Tragédies saintes (1566), éd. crit. C. Comte, Paris, E. Cornély (STFM), 1907, p. 23.

${ }^{34}$ Ibid., v. 415-417, p. 30.

${ }^{35}$ George Gifford, A Dialogue concerning Witches and Witchcrafts [...] ( $1^{\mathrm{e}}$ éd. 1593), Londres, 1603, reprint Londres, The Percy Society, 1842, p. 53 : «It is no small abhomination to go for helpe unto the divel: it is to set him in Gods place, and to honour him as God. It riseth of infidelitie and distrust of help from God, as we may see in the example of king Saule, who finding no answer nor comfort from God, whom he had so wickedly disobeyed, went to a witch. The heathen man said, Flectere si nequeo Superos, Acheronta movebo » 
$\operatorname{Dieu}^{36}$. Flavius Josèphe donne des précisions sur l'état physique de Saül en proie au démon:

Saul tomba en griefves passions, et devint demoniaque, en sorte qu'il sembloit qu'on l'estoufast ou estranglast $[\ldots]^{37}$.

Saül est présenté par Nider comme un «lunatique $»^{38}$. Nicolas de Lyre définit ainsi ce type de possession :

[...] on appelle lunatiques ceux qui, avec toutefois la permission divine, son affligés par les démons selon la disposition variable de la lune, car la lune domine sur les choses humides, et ainsi le cerveau de l'homme, qui est la partie la plus humide du corps humain, est plus apte à subir l'action du démon en un temps qu'en un autre, selon le mouvement variable de la lune $\mathrm{e}^{39}$.

Nider affirme que «Saül, désobéissant à Dieu, fut possédé ${ }^{40}$. C'est un cas de possession «pour grave crime personnel $»^{41}$, où «les démons exercent leur pouvoir sur les hommes, mais seulement pour autant que Dieu le leur permet ${ }^{42}$ " et «en châtiment, ils les privent pour un temps seulement de l'usage de la raison ${ }^{43}$ ». Selon Nider, si, lorsque David jouait de la cithare, l'esprit malin s'éloignait de Saül ${ }^{44}$, c'est que la musique guérissait celui-ci de sa disposition à subir l'action du démon. Nider cite Paul de Burgos (ca 1351-1435) :

Il ne s'accorde pas avec la lettre de dire que cela venait du mérite de David ou était le fait de ses prières ; car il n'est pas vraisemblable que l'Écriture s'en tairait, du moment que ce serait notoirement à la louange de David, chose dont pourtant elle ne fait nulle mention ${ }^{45}$.

${ }^{36}$ J. Calvin, Homilia LIX in I. Lib. Samuelis cap. XVI, CO, t. XXX, col. 177-180.

${ }^{37}$ Flavius Josèphe, éd. cit., p. 204.

${ }^{38} \mathrm{~J}$. Nider, Les sorciers et leurs tromperies (La Fourmilière livre V), 11.28, trad. J. Céard, Jérôme Millon, 2005, p. 206.

${ }^{39}$ Nicolas de Lyre, glose de I S 16, 23, éd. cit., f. cc2 $\mathrm{r}^{\circ}:$ " [...] unde et dicuntur aliqui lunatici [...], qui secundum variam dispositionem lune affliguntur a demonibus ex diuina tamen permissione: qua luna habet dominium super humida, et secundum hoc cerebrum hominis, quod inter omnes partes corporis est magis humidum, magis est aptum ad suscipiendum actionem demonis uno tempore quam alio, secundum varium motum lune.» (cité et traduit par J. Céard, in J. Nider, Les sorciers et leurs tromperies, éd. cit., n. 310 p. 266. ${ }^{40}$ J. Nider, op. cit., 11.19, p. 202 : «Saul inobediens Deo possessus».

${ }^{41}$ Ibid., 11.12, p. 200 : «pro magno facinore proprio». Voir H. Institoris, J. Sprenger, Le Marteau des Sorcières, Malleus Maleficarum (1486), II, 10, trad. A. Danet, Jérôme Millon, 1990, p. 331.

${ }^{42}$ J. Nider, op. cit., 11.22, p. 204 : «potestatem exercent in homines, sed tantummodo a Deo permissi fuerint $»$.

${ }^{43}$ Ibid., 11.23, p. 204 : «castigacione eorum ad tempus tantummodo usu rationis priuantur ». ${ }^{44}$ I S 16,23

${ }^{45}$ J. Nider, op. cit., 12.15-16, éd. cit., p. 226 : «Nec consonum est littere, dicere quod hoc fiebat ex meriti Dauid, seu per oraciones, quia non est verisimile, quod hoc scriptura taceret, cum esset in laudem Dauid notabiliter, de quo tamen nullam mencionem facit. » (trad. p. 227) 
Au contraire, Le Marteau des Sorcières, qui cite aussi le texte de Paul de Burgos ${ }^{46}$, explique le retrait de l'esprit malin par la présence de David, préfiguration du Christ :

Mais la raison pour laquelle l'esprit mauvais se retirait quand David jouait de la cithare, c'était à cause de la vertu de la Croix. On le dit assez expressément dans la Glose sur ce texte : David expert en musique, habile en sons et concerts harmonieux était signe de l'Unité de l'Essence qui chaque jour résonne sur des modes divers. Il repoussait l'esprit mauvais par la cithare, non parce qu'il y avait pareille vertu dans la cithare mais dans le signe de croix : sur le bois de la croix, les cordes des veines tendues, pendait Celui qui déjà chassait les démons ${ }^{47}$.

Pour Calvin, de même, la musique de David aurait été inefficace sans l'action de Dieu :

Ce n'a certes pas été la puissance de la cithare, ni celle de la mélodie ou des autres instruments de musique, qui auraient pu chasser et conjurer les diables; mais la volonté de Dieu a donné la puissance efficace pour faire obstacle à ceux-ci ${ }^{48}$.

Ainsi quatre caractéristiques majeures de Saül se dégagent: une tient à sa position historique - il a été un tyran -; les trois autres ressortissent à son idiosyncrasie: c'est un mélancolique, un envieux et un démoniaque. Nous nous concentrerons sur ces trois dernières, et nous déterminerons si ces figures sont indépendantes ou s'il est possible de les articuler.

À la Renaissance, la formule de saint Jérôme selon laquelle «la mélancholie est le bain du Démon» est assez souvent citée ${ }^{49}$. Dans son traité De Miraculis occultis natura, publié pour la première fois en 1559, Levinius Lemnius affirme que les maladies sont causées par les humeurs et non par les esprits malins, mais il concède que ceux-ci se mêlent à celles-là : « les malins esprits, par volonté divine, ou permission, se meslent parmi, et augmentent leur violence et fureur» et par conséquent «nous lisons Sathan avoir aigry la melancolie de Saul, et l'avoir incité à

${ }^{46}$ H. Institoris, J. Sprenger, Le Marteau des Sorcières, II, 5, éd. cit., p. 409-410.

${ }^{47}$ Ibid., I, 5, éd. cit., p. 171-172; id., Malleus maleficarum in Malleorum quorundam Maleficarum [...] tomi duo, Francfort, 1582, t. I, p. 90 : «Sed quod spiritus malus recederet quando Dauid cytharizabat, hoc erat propter uim crucis, quod satis expresse dicitur in glossa, ubi sic dicitur: Erat Dauid in canticis musicis eruditus, diuersorum sonorum rationabilis, moderatusque concentus. Unitatem essentia significat, qua variis modis quotidie resonat. Dauid malignum spiritum in cythara compescuit, non quia tanta esset uis in cythara, sed in signo crucis, qui in ligno crucis, et cordarum extensione, id est, uenarum gerebatur, qua iam tunc Damones effugebat. "

${ }^{48}$ J. Calvin, Homilia LIX in I Lib. Samuel, cap. XVI, 13-23, in C.O., t. XXX, col. 182 : «Sane non ea fuit vis cithara, non musices aut aliorum organicorum instrumentorum, ut pellere diabolos et coniurare possent : sed Dei voluntas efficaciam illis ad hoc officiendum dedit. "

${ }^{49}$ Par exemple, J. Wier, De Prastigiis Damonum, et incantationibus ac ueneficiis (écrit entre 1561 et $1562,1^{\mathrm{e}}$ éd. Bâle, 1564), IV, 25, Bâle, Oporin, 1577, col. 480 : «melancholiam esse diaboli balneum.» 
meurtres et trahisons et plusieurs choses malheureuses ${ }^{50} »$. De même, Jean Taxil affirme que la mélancolie est propice à l'influence du démon :

les corps que le Diable possede interieurement, sont melancholiques: car cest humeur est le vray siege, auquel le Diable se plait, et duquel il faict des effects si estranges $[\ldots]^{51}$.

Selon le médecin Jourdain Guibelet, c'est parce qu'il est couard que le mélancolique est prédisposé à subir l'action des démons :

[...] les possedez et demoniaques [...] sont recognus ordinairement ou de mauvaise vie, ou negligens au fait de la religion, ou debile d'esprit et de courage, $\square v \alpha v \delta \rho o 1$ selon S. Denys, qui dit qu'en tous possedez il y a soupçon d'une âme lasche, et de peu de courage. Au moyen dequoy, à mon advis, nous voyons plus de femmes que d'hommes estre possedées [...]. Pour cette mesme raison, les melancholiques sont volontiers possedez, dautant que cete humeur blesse l'imaginative, et imprime des fantasies tristes et pleines de facheries, d'où bien souvent s'ensuit le desespoir, qui est une des portes principales, pour donner entrée au Diable. Damon quoscumque superat per marorem superat (Johannes Chrysostomus, lib. 3 de providentia) ${ }^{52}$.

Sainte Thérèse (1515-1582) s'accorde avec les médecins lorsqu'elle écrit de la mélancolie :

Il est certain que le démon l'utilise pour essayer de se gagner certaines personnes; il y parviendra, si elles ne sont pas sur leurs gardes. Étant donné que cette humeur commence par assujettir la raison, une fois que celle-ci est obscurcie, que ne feront pas nos passions $^{53}$ ?

En sens inverse, Martin del Rio (1551-1608), qui déclare que «Saulem [...] daemon per atram bilem diuexebat ${ }^{54} "$, estime que le diable agit sur l'organisme en déclenchant des maladies mélancoliques :

En cette façon, il excite les maladies mélancoliques. Car, du commencement, il esmeut la bile noire, qui est dans le corps, et en pousse les fumées aux cellules des

\footnotetext{
${ }^{50}$ L. Lemnius, Les occultes merveilles et secretz de la nature, trad. fr. de J[acques] G[ohory], Lyon, 1574, f. $115 \mathrm{r}^{\circ}-\mathrm{v}^{\circ}$.

${ }^{51}$ J. Taxil, Traicté de l'epilepsie, Maladie vulgairement appellée au Pays de Provence, la gouttete aux petits enfans, Lyon, Robert Renaud, 1602, p. 157.

${ }_{52} \mathrm{~J}$. Guibelet, De l'Humeur melancolique, in Trois discours philosophiques, Evreux, Antoine Le Marié, 1603, f. $284 \mathrm{r}^{\circ}-\mathrm{v}^{\circ}$.

${ }^{53}$ Santa Teresa de Jesús, Libro de las fundaciones, I, 7, 2, in Obras completas, Madrid, BAC, 1986, p. 700 : «Cierto creo que el demonio en algunas personas le toma por medianero para si pudiese ganarlas, y, si no andan con gran aviso, sí hará; porque como lo que más este humor hace es sujetar la razón, ésta secura, ¿ qué no haran nuestras passiones?» (Trad. Paris, Desclée de Brouwer / 10/18, 1952, p. 53)

${ }^{54}$ M. Del Rio, Disquisitionum magicarum libri sex, III, V, Mayence, Petrus Henningius, 1617, p. 405 .
} 
sens interieurs. Puis après, il augmente ceste humeur par l'accés des choses brulantes, ou bien la retient et l'empesche de s'esvacuer ${ }^{55}$.

Pour les exorcistes, il est même difficile de distinguer entre la mélancolie qui résulte d'une dyscrasie naturelle et celle qui est causée par le diable :

[Le démon] s'allie à l'humeur mélancolique parce que c'est un milieu conforme à la nature des démons en raison de son amertume, de sa tristesse, et son trouble et si cette humeur était évacuée, le démon serait forcé de faire marche arrière. [...] D'ordinaire, le démon se cache très souvent dans les maladies naturelles dont nous avons parlé. Ainsi l'exorciste se demande avec embarras si la maladie a une origine diabolique ou naturelle, et il se trompe. De là vient le fait que parfois, le démon s'échappe impunément, parce que sa ruse n'est pas connue de l'exorciste ${ }^{56}$.

Ainsi, la démonologie renvoie à la physiologie des humeurs: la prédominance de l'humeur noire rend vulnérable aux tentations du démon et le diable provoque des accès de mélancolie.

La colère et l'envie se rapprochent de la mélancolie en ce qu'elles s'accompagnent de tristesse. D'une part, Thomas d'Aquin, reprenant Aristote, affirme que «l'action de la colère est accompagnée de tristesse ${ }^{57} »$. D'autre part, une longue tradition, qui remonte au moins à Damascène, définit l'envie comme "la tristesse que donne le bien d'autrui ${ }^{58}$ ». Thomas d'Aquin fait même de l'envie une des espèces de la tristesse. Comme toute tristesse, elle appesantit l'animus. L'aggravatio animi produite par la tristesse entrave le mouvement, s'oppose à la tension vers le plaisir. Elle a pour effet d'affaiblir toute opération humaine ${ }^{59}$.

L'envie est de tous les péchés celui qui semble lié le plus étroitement à l'influence du diable. Les théologiens répètent la formule du Livre de la Sagesse :

${ }^{55}$ M. Del Rio, Les Controverses et Recherches magiques, III, V, Paris, Jean Petit-Pas, 1611, p. 405 (cité par Jean Céard, «Entre le naturel et le démoniaque : la folie à la Renaissance» in Nouvelle Histoire de la Psychiatrie, sous la direction de Jacques Postel et Claude Quetel, Dunod, 1994, p. 89); id., Disquisitionum magicarum libri sex, éd. cit., p. 410: «Sic Melancholicos morbos excitat. Nam principio bilem atram, quae corpori inerat, commouet, et fuligines in cerebrum, et internorum sensuum cellulas propellit. Deinde adauget bilem atram, admotis causis exurentibus, vel detento isto humore, ne expurgetur. "

${ }_{56}$ Zacharia Vicecomes, Complementum artis exorcistica [...], in Thesaurus exorcismorum atque conjurationum terribilium, potentissimorum, efficacissimorum [...], Cologne, Lazarus Zetzner, 1608, p. $774:$ :[...] melancholico humore commiscetur quia Damonum natura sedes congrua est propter suam amaritudinem, tristitiam, et turbulentiam, qui humor si fuerit evacuatus Damon cogitur recedere. [...] In dictis naturalibus infirmitatibus, damon quam sepissime se occultare solet. Et sic Exorcista solet dubitare, an infirmitas sit diabolica, vel naturalis, et fallitur. Unde damon aliquando, non cognita ab Exorcista ejus astutia, impune evadit. »

${ }^{57}$ S. Th., I-II, q. 46, a. 2, obj. 3 : «ira operatur cum tristitia»; cf. Eth. à Nicomaque, VII, 1149 b 20, trad. J. Tricot, Vrin, 1967, p. 346: «tout homme agissant par colère agit en ressentant de la peine $(\lambda v \pi \mathrm{o} \square \mu \varepsilon v \mathrm{~s})$ ).

${ }^{58}$ Damascène, De Fide Orth., II, 14, PG 94, 932. Cf. Thomas d'Aquin, S. Th., II-II, q. 36, a. 2, obj. 2.

${ }_{59}$ S. Th., I-II, q. 37, a. 2-3. 
«C'est par l'envie du diable que la mort est entrée dans le monde. " ${ }^{60}$ Thomas d'Aquin commente ce passage en déclarant que «le démon, en suggérant l'envie, met en nous ce que lui-même a principalement dans le cœur. ${ }^{61}{ }^{6}$ Un médecin tel que Jean Wier prolonge ce discours. Dans l'édition augmentée qu'il donne en 1577 de son grand traité de démonologie, il ajoute une précision significative : les malins esprits ont trompé Eve «pour l'envie qu'ils portoyent à la felicité de l'homme ${ }^{62}$ ». Wier compte les envieux parmi les personnes les plus vulnérables aux démons.

Le diable est aux embusches apres telles gens, le plus qu'il peut, et par tous moyens, observant tousjours le lieu et le temps; et selon qu'il conoit les personnes par certains signes et indices, estre adonnez ou affectionnez naturellement, il les aborde par quelque raison particuliere, il les poursuit, il les alleche $[\ldots]^{63}$.

Plus encore que d'envie, Saül est coupable de désespoir. La glose de Nicolas de Lyre compte parmi ses fautes la perte de l'espoir en Dieu ${ }^{64}$. Pour Calvin, Saül se livre au démon parce qu'il est désespéré d'avoir été abandonné par Dieu :

[...] quand on ronge son frein, et qu'on s'opiniastre en sa fascherie, jusques à venir en desespoir, qu'on ne veut plus recevoir nulle consolation, qu'on repousse toute memoire de Dieu, et voudroit-on que son nom fust enseveli : alors Satan a la porte ouverte, et viendra faire ses illusions, et n'y peut-on resister. Et nous avons en cela un beau miroir en Saul. Car il avoit offensé Dieu : il faut qu'il soit despouillé du royaume, puis que le plaisir de Dieu est tel: il n'y a nul moyen, sinon qu'il s'humilie. Or il ne le veut point faire. Et quand il voit qu'il n'y a nul moyen d'appaiser Dieu: et qu'après avoir flatté Samuel, tant s'en faut qu'il y profite, qu'apres qu'il a desciré le manteau du Prophete, il luy est dit: Dieu dissipera ainsi ton royaume. Il crie, il se jette hors des gonds. Et que fait-il plus? O puis que Dieu me delaisse, il faut que je le quitte aussi de mon costé. Et quelle est l'issue? Il s'en va apres les sorciers ${ }^{65}$.

Dans la pièce que Jean de la Taille consacre à Saül, l'écuyer dit au roi :

\footnotetext{
${ }^{60} \mathrm{Sg} 2,24:$ : Inuidia autem diaboli mors introiuit in orbem terrarum. »

${ }^{61}$ S. Th., II-II, q. 36, a. 4, sol. 2 : «[...] quando diabolus invidiam suggerit, ad hoc hominem inducit quod ipse principaliter in corde habet.»

${ }^{62}$ J. Wier, De Prastigiis Damonum, et incantationibus ac ueneficiis (écrit entre 1561 et 1562, $1^{\mathrm{e}}$ éd. Bâle, 1564), I, 3, Bâle, Oporin, 1577, col. 24 : «alience felicitatis inuidia » (Histoires, Disputes et Discours, des illusions et impostures du diable [...], I, 3, [Genève], Jacques Chouet, 1579, p. 7).

${ }^{63}$ Ibid., III, 5, col. 251 : «His ut instrumentis congruis insidiatur quibuscumque potest modis, suo tempore et loco: singulos, ut cuiusque animi studia et affectus ex certis indiciis cognouerit, peculiari aliqua ratione adoritur, insectatur, illicit [...].» (trad. cit. p. 219)

${ }^{64}$ Voir Nicolas de Lyre, glose de I S 31, 5, éd. cit., f. dd $3 \mathrm{v}^{\circ}$ : «insuper etiam pythonissam consuluerit nec sperauerit in domino " (en outre il aura même consulté la pythonisse et il n'aura pas espéré en Dieu).

${ }^{65}$ J. Calvin, Sermon sur Deut. XVIII, 10-15 [1555], in CO, LV, p. 510-511, cité par E. Forsyth, in J. de La Taille, Saül le Furieux, La Famine, ou les Gabeonites, Paris, Marcel Didier, 1968, p. 39.
} 
Ne vous deseperez, mais avec fiance

Et bon espoir prenez vos maux en patience $[\ldots]^{66}$.

Le désespoir de Saül est particulièrement sensible dans une pièce comme David combattant, où, face aux rodomontades de Goliath, Saül reste prostré, indécis, paralysé par la peur; parce qu'il ne croit pas à l'aide divine, son entourage doit l'exhorter à reprendre courage ${ }^{67}$ ou au moins à faire semblant ${ }^{68}$; alors que David "Toujours en Dieu est esperant ${ }^{69}$ » et puise dans sa foi l'énergie qui assure la victoire.

Ainsi, aux $\mathrm{XV}^{\mathrm{e}}$ et $\mathrm{XVI}^{\mathrm{e}}$ siècles, un réseau de correspondances secrètes se tisse entre les figures du tyran, du mélancolique, de l'envieux et du possédé : si parfois l'une de ces causes de la colère de Saül est mise en doute, les quatre interprétations ne paraissent aucunement incompatibles. On ne peut même pas considérer qu'elles relèveraient en propre de disciplines distinctes, tant les savoirs se pénètrent l'un l'autre. Il n'est pas surprenant que le théâtre se nourrisse de discours spécialisés, qu'il les amalgame et les fusionne; mais les discours de la philosophie politiques, de la médecine, de la théologie morale, de la démonologie, si techniques qu'ils soient, ne sont pas étanches. Chez ceux qui les tiennent, nulle crainte de s'aventurer hors de leur champ d'investigation, nul souci de défendre un terrain de compétence légitime, nul besoin non plus d'exclure la parole exogène. Au contraire, le discours technique est naturellement hégémonique. Toute savoir particulier tend à proposer une interprétation totale. En effet, les hommes de la fin du Moyen Âge et de la Renaissance postulent l'unité fondamentale de l'être, et conçoivent les sciences comme des moyens complémentaires d'observer la Création et de guetter à travers elle l'acte divin.

Or chacun, lorsqu'il sort de son domaine, est tributaire de la phraséologie et des méthodes de celui dans lequel il s'immisce. Les théologiens ne disposent pas d'autres catégories pour appréhender les comportements pathologiques que celles que leur fournit la médecine d'Hippocrate et de Galien. De même, les médecins assimilent les acquis de la démonologie: s'il arrive à Jean Wier de proposer une interprétation psychologique des phénomènes de possession, il ne nie pas l'action du diable. La pensée de la Renaissance se refuse à définir des parcelles de savoir rigoureusement bornées, à concevoir la connaissance comme un paysage de champs clos.

Cet esprit de conciliation a néanmoins des limites. Il vaut tant qu'il ne menace pas la légitimité du discours. Le frontispice des ouvrages scientifiques rappelle les fonctions officielles de l'auteur: Jean Nider est «Professeur de théologie », Jean Wier est "médecin du Duc de Clèves », Martin del Rio est "Prêtre de la société de Jésus, licencié en droit, docteur en théologie de longue date»... L'autorité de la parole savante risquerait de diminuer si la situation d'énonciation ainsi créée n'était pas pleinement assumée.

${ }^{66}$ J. de La Taille, Saül le Furieux (1573), II, v. 405-406, éd. cit., p. 38.

${ }^{67}$ L. Des Masures, David combattant, v. 418-433, in Tragédies saintes, éd. cit., p. 30-31.

${ }^{68}$ Ibid., v. 905-908, p. 51-52.

${ }^{69}$ Ibid., v. 1621 , p. 84. 
Dans son domaine propre, le spécialiste pratique l'assertion et parfois même la polémique; lorsqu'il sort de son champ de compétence, il adopte une position prudente et consensuelle. Si un médecin parle du démon, il veille à ne pas froisser les théologiens: on se montre d'autant moins critique envers une tradition que l'on s'en sait moins tributaire. Dans le traité De Sacra Philosophia, publié pour la première fois en 1588, Francisco Valles, médecin-chef de Philippe II, fait à propos de la Bible des observations de philosophie naturelle. Il commente en particulier le passage du premier Livre de Samuel qui reproduit les paroles des serviteurs de Saül : «Voici qu'un esprit mauvais, venu de Dieu, te tourmente. Que notre Seigneur parle. Tes serviteurs sont à ta disposition : ils chercheront un homme qui sache jouer de la cithare ; ainsi, quand un esprit mauvais, venu de Dieu, t'assaillira, il en jouera et cela te soulagera. ${ }^{70}$ Valles rapporte d'abord l'interrogation des théologiens et la façon dont elle est d'ordinaire tranchée :

En vérité, si Saül n'était pas tourmenté par l'humeur mélancolique, mais par le Démon, quelle pourrait être la raison pour laquelle il était égayé par les vibrations de la cithare et supportait mieux ce tourment? Est-ce que le Démon était adouci par la musique? Devant cette incertitude, le plus grand nombre pense qu'il n'y a rien eu ici de naturel, mais que cela a été produit par une force divine, de sorte que lorsque David jouait de la cithare, le Démon était chassé, non tant à cause de la cadence du son, qu'à cause des hymnes divins qu'il prononçait en chantant. C'est pourquoi il chasse le Démon par les divins psaumes, comme par des exorcismes ${ }^{71}$.

Il s'agit là d'une concession faite aux théologiens, mais Valles s'empresse de démontrer que les causes profondes du malaise de Saül, qu'elles soient naturelles ou démoniaques, sont indifférentes pour le médecin, qui ne connaît que la cause immédiate, la mélancolie. Il poursuit en ces termes :

Assurément, pour ma part, je ne nie pas cela, et je ne doute pas que par ce procédé, la force du verbe de Dieu et de la prophétie de David ait été signifiée; je pense néanmoins que la cause naturelle s'est conjuguée avec cette cause divine et que cet effet étonnant doit être rapporté à l'une et à l'autre. J'indiquerai facilement cela, après avoir exposé au regard de tous certaines paroles d'Avicenne, dans son chapitre 18 , première fen. de son quatrième traité qui traite de la mélancolie, ce sont : «Et il a semblé à certains des médecins que la mélancolie est produite par le démon; mais nous ne nous soucions pas, lorsque nous enseignons la médecine, si cela est produit par le démon ou non, dès lors que nous avons dit que, si cela est produit par le démon, c'est dans la mesure où il fait tourner le tempérament à la bile noire, et où la

\footnotetext{
${ }^{70}$ I S 16, 15-16, Traduction Ccuménique de la Bible, Le Cerf, 1975.

${ }^{71} \mathrm{~F}$. Valles, De iis qua scripta sunt physice in libris sacris, sive de sacra philosophia $\left(1^{\mathrm{e}}\right.$ éd. Lyon, 1588), XVIII, Lyon, Hugues de La Porte, chez les frères de Gabiano, 1592, p. 220 : «Si uero Saul non humore agitabatur melancholico, sed Damone, qua causa esse possit, ut cythara pulsatione iunaretur? et leuius eam exagitationem ferret? An Damon mitescit musica? Hanc ob dubitationem, plurimi nihil hic censent fuisse naturale, sed diuina ui effectum, ut pulsante Davide cytharam abigeretur Damon, non tam ob soni numeros, quam ob diuinos hymnos quos ille cantando recitabat. Itaque diuinis Psalmis, ut exorcismis abigit Damonem.»
} 
cause immédiate de ceci est la bile noire, que la cause de cela soit ensuite le démon ou non ${ }^{72}$.»

La citation d'Avicenne est assez claire : le démon est pour le médecin une hypothèse inutile. Plus loin, Valles est encore plus net en déclarant que «le démon a la fonction d'une cause externe ${ }^{73} »$.

L'exemple de Valles enseigne qu'un second facteur contribue à l'individualisation des savoirs: leur finalité pratique. Le penseur politique, le médecin, le confesseur, l'exorciste, conçoivent la connaissance comme le moyen d'une action sur le réel. Soucieux d'efficace, ils se hasardent parfois hors de leur territoire naturel, mais l'objectif que chacun s'assigne - la définition de la monarchie, la santé du patient, la pénitence du fidèle, la lutte contre démon - établit entre les disciplines une ligne de démarcation.

Ainsi, l'imbrication des discours n'est pas leur indistinction: chaque discipline pose son objet propre et à partir de celui-ci établit une hiérarchie des discours qu'elle accueille, et une spécialisation s'opère.

Dès lors que l'on considère le dialogue qui, au Moyen Âge et à la Renaissance, s'instaure entre les savoirs, la colère de Saül n'est plus aussi étrange qu'elle le semblait de prime abord. La pensée de l'époque multiplie les approches ; elle ne trie pas les causes, elle les accumule; et pourtant les discours ne se juxtaposent pas dans un rapport de contiguïté : une exigence de cohérence globale préside à toute hypothèse nouvelle; l'ensemble des explications s'organise selon une syntaxe secrète, qui évite la disparate et la contradiction. Le secret de cette harmonie est l'hybridation des discours. À l'éclectisme philosophique, que certains considèrent comme une caractéristique des $\mathrm{XV}^{\mathrm{e}}$ et $\mathrm{XVI}^{\mathrm{e}}$ siècles, répond un syncrétisme épistémologique.

Bruno Méniel

Université de Rennes 2-CELAM

\footnotetext{
${ }^{72}$ Ibid., p. 220-221 : «Ego quidem hoc non nego, neque dubito illa ratione, vim verbi Dei, et Dauidica Prophetia significatam esse: nihilominus censeo, naturalem quoque causam cum diuina illa coniunctam esse, atque admirandum illum effectum, in utramque referendum fuisse. Hoc indicabo facile, productis in medium quibusdam Auicenna uerbis, ex capite 18. fen. prima, tractatus quarti quod est de Melancholia, ea uero sunt hac: Et quibusdam Medicorum uisum est, quod melancholia contingat a Dæmonio, sed nos non curamus, cum Physicam docemus, si illud contingat a Dæmonio, aut non contingat, postquam dicimus, quod si contingat a Dæmonio, tunc contingit ita ut conuertat complexionem ad choleram nigram, et sit causa eius propinqua cholera nigra, deinde sit causa illius Dæmonium, aut non Dæmonium. »

${ }^{73}$ Ibid., p. 223 : «habetque Damon uicem causa externa ».
} 